\title{
Pedometer step counting in South Africa: tools or trinkets?
}

\author{
lan Cook (BA (Phys Ed) Hons, BSc (Med) Hons) \\ Department of Kinesiology and Physical Education, University of Limpopo, Polokwane
}

\begin{abstract}
Objectives. This study addressed ( $i$ ) the accuracy of measuring ambulatory signals and (ii) the susceptibility to nonambulatory signals, of the Discovery Vitality Pedometer (VT) and the Kellogg's Special K Step Counter (KL) compared with three research-grade pedometers (DW: Yamax DigiWalker SW-401, MTI: MTI Actigraph AM-7164-2.2 , NL: New Lifestyles NL 2000).

Design. One hundred instruments (20 instruments/brand) were tested at five level walking speeds on a motorised treadmill $\left(3.24,4.02,4.80,5.64,6.42 \mathrm{~km} \mathrm{hr}^{-1}\right)$ and during motor vehicle travel on tarred roads $(62.9 \mathrm{~km})$.
\end{abstract}

Results. The $\mathrm{KL}$ was highly variable across all speeds, while the VT tended to be variable at the lowest speed. The DW, NL and VT significantly underestimated steps below $4.80 \mathrm{~km} . \mathrm{hr}^{-1}(41-94 \%, p<0.02)$ but accuracy improved at speeds $\geq 4.80 \mathrm{~km} \cdot \mathrm{hr}^{-1}(98-102 \%)$. The KL displayed the highest variability $(60 \%$ inter-instrument variance) followed by the VT ( $10 \%$ inter-instrument variance). The research-grade pedometers were the least variable (0 - 1\% inter-instrument variance). At $4.80 \mathrm{~km} . \mathrm{hr}^{-1}$, all research-grade pedometers measured within a $10 \%$ margin of error compared with the $90 \%$ of VT units and $42 \%$ of $\mathrm{KL}$ units. The VT was significantly more resistant to nonambulatory signals than the DW $(p<0.01)$. The $\mathrm{KL}$ was the most variable in its response to non-ambulatory signals while the NL was the most consistent. The MTI detected the most non-ambulatory signals $(p<0.05)$.

Conclusions. The $\mathrm{KL}$ should not be used as a promotional pedometer. The VT achieved the minimum standards required of a promotional pedometer. Further testing is

\section{CORRESPONDENCE:}

Ian Cook

Department of Kinesiology and Physical Education

University of Limpopo (Turfloop Campus)

PO Box 459

Fauna Park

0787

Polokwane

Tel+fax: +27 152682390

E-mail: ianc@ul.ac.za required for longevity, and performance under free-living conditions.

\section{Introduction}

Obesity is a global $^{15}$ and local ${ }^{30}$ epidemic and is associated with numerous comorbidities, ${ }^{28}$ such that cardiovascular disease, hypertension and diabetes are a global and regional threat. $^{17,45,47}$ There seems to be agreement that the successful implementation of public health initiatives will require some degree of social responsibility from private industry. ${ }^{42,46}$ For instance, some fast-food chains have adjusted their products to cater for healthier food choices and have included step counters or pedometers in their product mix to promote greater public awareness of the importance of a physically active lifestyle and to improve their public image. ${ }^{13}$

Nationally, several major companies have launched campaigns in 2005 using pedometers or step counters to promote an active lifestyle. In 2005, Kellogg's South Africa launched the Kellogg's Special K Step Counter (KL), ${ }^{19}$ and Discovery Health introduced the Vitality Pedometer (VT) as part of their Vitality programme. ${ }^{12}$ These South African initiatives are in agreement with international health promotion programmes as seen in Australia, ${ }^{1}$ Europe $^{41}$ and North America. ${ }^{3}$ The Kellogg's promotion encourages pedometer users to increase their daily ambulatory activity by 2000 steps over and above their usual ambulation level, ${ }^{19}$ while Discovery Health launched a similar 10000 steps programme. $^{12}$

Although the marketing initiatives of Kellogg's South Africa and Discovery Health are laudable, the accuracy and longevity of the pedometers are not reported. Calibration studies have found that the accuracy,9,21,22,25,32 and longevity ${ }^{24}$ of pedometers vary considerably between brands. Recent validation trials of promotional pedometers in Europe $^{10}$ and North America ${ }^{14,37}$ have found poor instrument accuracy and consistency. These studies concluded that poor quality promotional pedometers detract from the public health message of a physically active lifestyle. ${ }^{10,14,37}$

It is important to note that the potential for substantial error and misclassification is possible if the pedometers are found to be inaccurate and unreliable or fragile. Erroneous pedometer readings could have a significant effect on the awarding of 'points' or incentives in the Discovery Vitality 
Lifestyle Programme $^{12}$ and Kellogg's Special K Challenge. ${ }^{19}$ For instance, with an $80 \%$ accuracy level, 10000 steps.day $^{-1}$ could be measured as 8000 steps.day $^{-1}$ or 12000 steps. day $^{-1}$. In other words, a participant could be classified as low active or highly active for the same pedometer reading. ${ }^{34}$ Customer reviews of the Discovery VT have reported wide discrepancies between pedometers ranging from 5000 steps to more than 40000 steps. One customer review reported the pedometer recording approximately 1 out of every 25 steps ( $4 \%$ accuracy level). ${ }^{11}$

Therefore, the objectives of this study were firstly to determine the accuracy of measuring ambulatory signals, and secondly to evaluate the susceptibility to non-ambulatory signals, of the VT and the $\mathrm{KL}$ pedometers as opposed to research-grade pedometers.

\section{Methods}

\section{Study design}

Five brands of pedometers, of which three are researchgrade instruments, were tested while:
- walking at five level speeds on a motorised treadmill, - and during motor vehicle travel on tarred roads.

For each pedometer brand 20 units were tested so that 100 pedometers were tested in total. For the purposes of this investigation a research-grade pedometer was defined as an instrument that has been found to be valid and consistent in its measurement of ambulatory activity, and as such is routinely used in descriptive and experimental studies which investigate aspects of physical activity and health. Promotional pedometers are defined as instruments that sporadically appear in the public domain through corporate initiatives but have not undergone any form of quality control conducted either by the corporate or an independent institution prior to distribution.

The study was approved by the Ethics Committee of the University of Limpopo (Turfloop Campus) and signed informed consent was obtained from subjects prior to participation in the study.

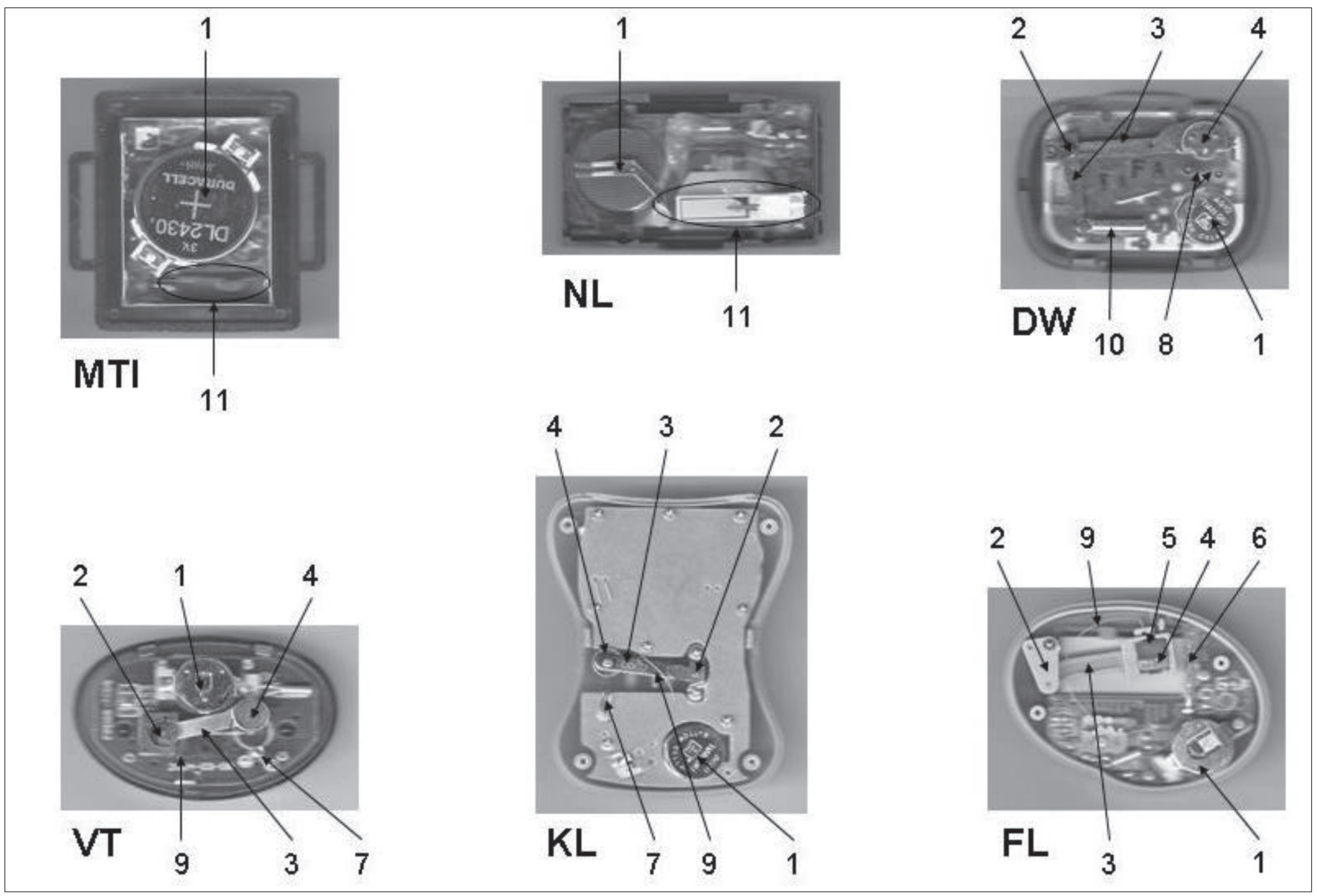

Fig. 1. Internal details of the step-sensing mechanisms of research-grade and promotional pedometers. $M T I=M T I$ Actigraph AM-7164-2.2, NL = New Lifestyles NL 2000, DW = Yamax DigiWalker SW-401, VT = Discovery Vitality Pedometer, KL = Kellogg's Special K Step Counter, FL = Flora Comrades Step Counter (the FL pedometer could not be obtained in time for the completion of the study, but it is included for comparative purposes, see discussion).

Key to internal details: 1 = battery, 2 = axis about which lever arm turns, $3=$ lever arm, $4=$ mass, $5=$ magnet, $6=$ magnetic reed switch, 7 = metal contacts, $8=$ dampened contacts, $9=$ hair spring, $10=$ coiled spring, $11=$ uni-axial, piezo-electric accelerometer mechanism (horizontal cantilevered beam with a mass on the end, piezo-electric crystal). 


\section{Pedometers}

The validity, reliability or longevity, construction and rating of these or similar pedometers have been studied and reviewed extensively elsewhere. ${ }^{33,43}$ As a qualitative assessment of pedometer quality, ${ }^{33}$ Fig. 1 details the internal construction of the pedometers.

The $\mathrm{KL}(6.4 \mathrm{~cm} \times 4.8 \mathrm{~cm} \times 2.2 \mathrm{~cm}, 21 \mathrm{~g})$ uses a hair spring and lever arm (Fig. 1) but does not incorporate any algorithms within the microprocessor to exclude non-ambulatory signals. The control buttons are not covered, raising the possibility of inadvertently resetting or stopping the unit. The South African promotion is based on a similar promotion in the United Kingdom. ${ }^{18}$ The pedometer was obtained from Kellogg's South Africa. ${ }^{19}$

The VT $(2 \mathrm{~cm} \times 5.3 \mathrm{~cm} \times 3.6 \mathrm{~cm}, 16 \mathrm{~g})$ can be obtained from Discovery Vitality partners ${ }^{12}$ or online. ${ }^{11}$ The VT step-counting mechanism also uses a hair spring and lever arm (Fig. 1). The VT incorporates an algorithm within the microprocessor to filter signals so as to exclude non-ambulatory signals. Because the control buttons are not covered the possibility exists of inadvertently resetting the unit.

The Yamax DigiWalker (DW, model SW-401, New Lifestyles Inc., MO, USA) ( $5 \mathrm{~cm} \times 3.8 \mathrm{~cm} \times 2 \mathrm{~cm}, 21 \mathrm{~g})$ uses a coiled-spring mechanism and lever arm to detect steps (Fig. 1). The DW does not utilise an algorithm to filter signals for non-ambulatory movement. A cover over the control buttons prevents the unit from being inadvertently reset or stopped. The DW is considered accurate ${ }^{5,6,9,21,32}$ and durable, ${ }^{24}$ and has been used for large-scale surveys $s^{4,36}$ and in experimental trials. ${ }^{16,27}$ The DW is a popular brand of pedometer and has been used in public health initiatives similar in concept to the Kellogg's and Discovery initiatives. ${ }^{1}$

The New Lifestyles NL 2000 (NL, New Lifestyles Inc., MO, USA) is slightly larger and heavier than the DW $(1.9 \mathrm{~cm} \times 3.5$ $\mathrm{cm} \times 5.7 \mathrm{~cm}, 31 \mathrm{~g}$ ). Instead of a spring mechanism, the $\mathrm{NL}$ pedometer consists of an accelerometer-type mechanism (Fig. 1). An algorithm in the microprocessor is used to filter the signal from the piezo-electric crystal in order to exclude non-ambulatory movement. The control buttons are covered, preventing the unit from being inadvertently reset or stopped. The NL is considered one of the most sophisticated pedometers marketed for public use and has been shown to have accuracy comparable to that of research-grade uniaxial accelerometers. ${ }^{9,31,32}$ As with the KL, VT and DW, the output (total number of steps recorded) for the NL is read off a small LCD screen.

The MTI Actigraph (MTI, model AM-7164-2.2, MTI Health Services, FL, USA) (5 cm x $4 \mathrm{~cm} \times 1.5 \mathrm{~cm}, 43 \mathrm{~g})$ is a research-grade movement monitor ${ }^{44}$ incorporating a uni-axial accelerometer and appropriate signal filters (Fig. 1). The MTI pedometer mode has been used as a criterion for pedometer validation ${ }^{21,22,44}$ and in experimental trials. ${ }^{20}$ There are no external buttons and the monitor output cannot be directly viewed but must be downloaded to an IBM compatible computer via an interface unit for further analysis using appropriate software (MTI Actisoft Analysis Software for Windows version 3.2). The number of steps recorded for the particular epoch period, e.g. total number of steps in 1 minute, is used to determine the total number of steps over the period of recording.

\section{Study protocol}

\section{Part 1. Accuracy}

The accuracy with which the pedometers (DW, KL, MTI, NL, VT) could measure 100 steps was evaluated on a calibrated motorised treadmill (Johnson JET-7000). One female subject (37 years, body mass: $65.0 \mathrm{~kg}$, stature: $165 \mathrm{~cm}$ ) wore pedometers (DW, KL, MTI, NL, VT) on the left side during three trials (the left side was chosen for ease of measurement during the trials, and because the pedometers were not all available at the same time, three trials had to be conducted). Each trial consisted of 20 walking bouts, and 1 walking bout included five speeds $\left(3.24,4.02,4.80,5.64,6.42 \mathrm{~km}^{-\mathrm{hr}^{-1}}\right.$ or 54, 67, 80, 94, $\left.107 \mathrm{~m} \cdot \mathrm{min}^{-1}\right)$. Corresponding approximate energy expenditures for these walking speeds is 2.5 METs, 3.0 METs, 3.3 METs, 4.0 METs and 5.0 METs, respectively (metabolic equivalents, $1 \mathrm{MET}=1 \mathrm{kcal} . \mathrm{kg}^{-1} \cdot \mathrm{min}^{-1}$ ). ${ }^{2}$ This protocol is analogous to testing all the pedometers on a single mechanical calibration rig $^{7,26}$ and is in agreement with the 'longevity' protocol of McKenzie et al. ${ }^{24}$ that used one subject walking on a treadmill, over a number of trials.

For the first trial, the MTI and the DW were placed over the midline of the thigh and the $\mathrm{KL}$ monitors were placed approximately $15 \mathrm{~cm}$ to the left of the central line of the waist as per manufacturers' instructions. Thus starting from the midline of the left thigh, the order of pedometer placement from back to front was MTI, DW and KL. For the second and third trials, the subject wore the VT and NL, respectively, over the centre of the left thigh as per manufacturers' instructions. The placement of the DW/MTI combination was such that the pedometers were positioned directly next to each other, with the midline of the thigh separating the two pedometers. Both pedometers were $\sim 1 \mathrm{~cm}$ from the midline of the thigh. There are no data to suggest that this distance from the midline of the thigh could affect pedometer output. In fact, some studies have placed the DW and MTI concurrently on the left and right side during treadmill validation trials. ${ }^{21}$ The pedometers were securely attached to a nylon belt which clipped around the subject's waist. The subject walked at five speeds (3.24, $4.02,4.80,5.64,6.42 \mathrm{~km} . \mathrm{hr}^{-1}$ ) with one or more pedometers, after which a new pedometer was attached to the subject's waist and the five-speed calibration process was repeated. The walking speeds were not randomised but applied in ascending order starting at the lowest speed.

The subject was well experienced in walking and running at low and fast speeds on a motorised treadmill. In addition, the subject was trained to step on or off the moving treadmill belt quickly and to stand quietly until instructed to step onto the treadmill belt again. The subject was also habituated to using the treadmill safety rails as little as possible when 
stepping onto the treadmill belt. For each trial the treadmill belt was adjusted to the proper speed while the subject stood quietly at the side of the treadmill. At the command of the investigator, the subject stepped onto the treadmill belt and started walking. Using a tally counter, 100 steps were counted. The last step was taken so that the subject stepped off the treadmill belt and stood quietly for at least 20 seconds while the treadmill speed was adjusted for the next walking speed and the readings from the pedometers (DW, KL, NL, VT) were recorded and the units reset. Prior to the first calibration session the MTI pedometers were activated and the recording epoch set at 5 seconds. Before each calibration walk, the subject stood quietly on the treadmill for 20 seconds to ensure a 'washout' prior to the next calibration walk. After the full calibration session, the data were downloaded from the MTI units to an IBM-compatible computer via an interface unit for further analysis. The accuracy of the pedometers to measure 100 steps was expressed as a percentage (100 steps $=100 \%$ ). A spirit level was used to ensure that the treadmill was level. The treadmill speed was calibrated before and after each calibration session by measuring the belt length $(3.305 \mathrm{~m})$ and the time it took to complete 25 revolutions at five speeds $\left(3.24,4.02,4.80,5.64,6.42 \mathrm{~km} . \mathrm{hr}^{-1}\right)$.

\section{Part 2. Susceptibility to non-ambulatory signals}

To evaluate the effect of motor vehicle travel on pedometer output, 100 pedometers (20 instruments from 5 pedometer brands; DW, KL, MTI, NL, VT) were randomly fastened to a custom-made wooden rig (width: $74 \mathrm{~cm}$ x length: $44.5 \mathrm{~cm} \mathrm{x}$ height: $9.5 \mathrm{~cm}$, mass: $3.7 \mathrm{~kg}$ ) along 10 columns and 10 rows. The rig was placed on the rear seat of a light motor vehicle (Ford Ikon 1.6i LX, 2004 model) and centered along the longitudinal axis of the motor vehicle. A $50 \mathrm{~kg}$ dead weight was placed on top of the wooden rig to prevent undue movement and vibration. The front passenger seat was unoccupied. Prior to the ride the pedometers were reset $(\mathrm{DW}, \mathrm{KL}$, $\mathrm{NL}, \mathrm{VT}$ ) and activated (MTI, 1 minute recording epoch). The trip was conducted in and around a metropolitan area on tarred roads to simulate usual driving patterns (variable and constant speed). After the ride the data (total number of steps) were recorded (DW, KL, NL, VT) or downloaded (MTI) to an IBM-compatible computer via an interface unit for further analysis. The total number of steps recorded during the ride were divided by the total distance covered $(\mathrm{km})$ and expressed as steps. $\mathrm{km}^{-1}$.

To obtain an accurate measure of the trip distance and speed a Garmin GPSMAP 76S handheld global position satellite (GPS) unit and an externally mounted (roof) antenna (Garmin GA 27C) were used. To ensure accurate positional readings the WAAS (Wide Angle Augmentation System) capability of the GPS unit was enabled which ensured a positional accuracy of $<2 \mathrm{~m}$. The position, distance and speed were recorded and stored automatically every second and downloaded via an interface cable to an IBM-compatible computer for further analysis using appropriate software (MapSource for Windows version 6.3).

\section{Statistical analysis}

Data are expressed as mean (SD) unless otherwise stated. For skewed continuous data, the median (interquartile range) is reported. Categorical data are expressed as a percentage.

\section{Part 1}

A general linear model (repeated-measures analysis of variance (ANOVA)) was used to determine if the pedometer readings differed across walking speeds. To examine differences between pedometers for each of the walking speeds a one-way ANOVA was used. Post hoc analyses for both the repeated-measures ANOVA and one-way ANOVA were performed using the Sidak correction for multiple comparisons to adjust the significance level.

To evaluate the sources of variability in pedometer data, variance components in random effects models were estimated using restricted maximum likelihood methods. ${ }^{23}$ Pedometer output (steps) was the dependant variable for this analysis. Variance components were estimated for pedometer variance (inter-instrument), trial variance (walking trial), and residual or error variance (intra-instrument). Trial variance and residual variance were nested within intra-instrument variance. Note that instrument refers to instruments of the same pedometer make. The variance components were also expressed as a percentage of the total variance. Interinstrument variance represents true variation between instruments while intra-instrument variance represents trialto-trial variation within instruments.

Pedometer step-counting accuracy at a walking speed of $4.8 \mathrm{~km} \cdot \mathrm{hr}^{-1}$ (3.3 METs) was computed using the following formula, per cent error $=$ (steps detected - actual steps $) /$ actual steps $\times 100$ ), and expressed as an absolute and relative value. $^{21,37} \mathrm{~A}$ margin of error of $10 \%$ was used as a minimum standard for a promotional pedometer. ${ }^{37}$ To categorise the direction of per cent error the following groupings were used; under-counting $(<-10 \%)$, exact $( \pm 10 \%)$ and over-counting $(>+10 \%)^{37}$

\section{Part 2}

Because the $\mathrm{KL}$ data were highly skewed data, the KruskalWallis test was used to examine differences across pedometers, and if significant, Dunn's multiple comparison test was used to determine which differences were significant.

A general linear model (univariate ANOVA) was used to determine if the placement of the pedometers on the rig was a significant factor (row and column effects). Because the $\mathrm{KL}$ data were highly skewed, all the pedometer data (steps. $\mathrm{km}^{-1}$ ) were ranked prior to running the statistical procedure. ${ }^{29}$

Significance for all inferential statistics was set at $p<$ 0.05. Data were analysed using SPSS for Windows 13.0 (Descriptive statistics, One-way ANOVA, General Linear Models, Variance Components) and GraphPad Prism 4.03 (Kruskal-Wallis and Dunn's Multiple Comparison) 


\section{Results}

\section{Part 1}

Because one $\mathrm{KL}$ pedometer malfunctioned (battery failure), data are reported for $19 \mathrm{KL}$ pedometers. For all other pedometers, data for 20 units per pedometer brand are reported.

In contrast to the research-grade pedometers (Figs $2 \mathrm{~A}-$ C), the VT pedometer (Fig. 2 D) was not consistent at lower walking speeds, but revealed far less variability than the $\mathrm{KL}$ pedometer (Fig. $2 \mathrm{E}$ ). The amount of variability between $\mathrm{KL}$ monitors over all the walking speeds, but especially at the lower walking speeds, is visually evident (Fig. 2 E). Most of the pedometers (DW, NL, VT) tended to significantly undercount the actual number of steps at the two lower speeds to varying degrees $(p<0.02)$ (Fig. 2, Table I). A threshold is evident at $4.80 \mathrm{~km} \cdot \mathrm{hr}^{-1}$; above this threshold four of the pedometers

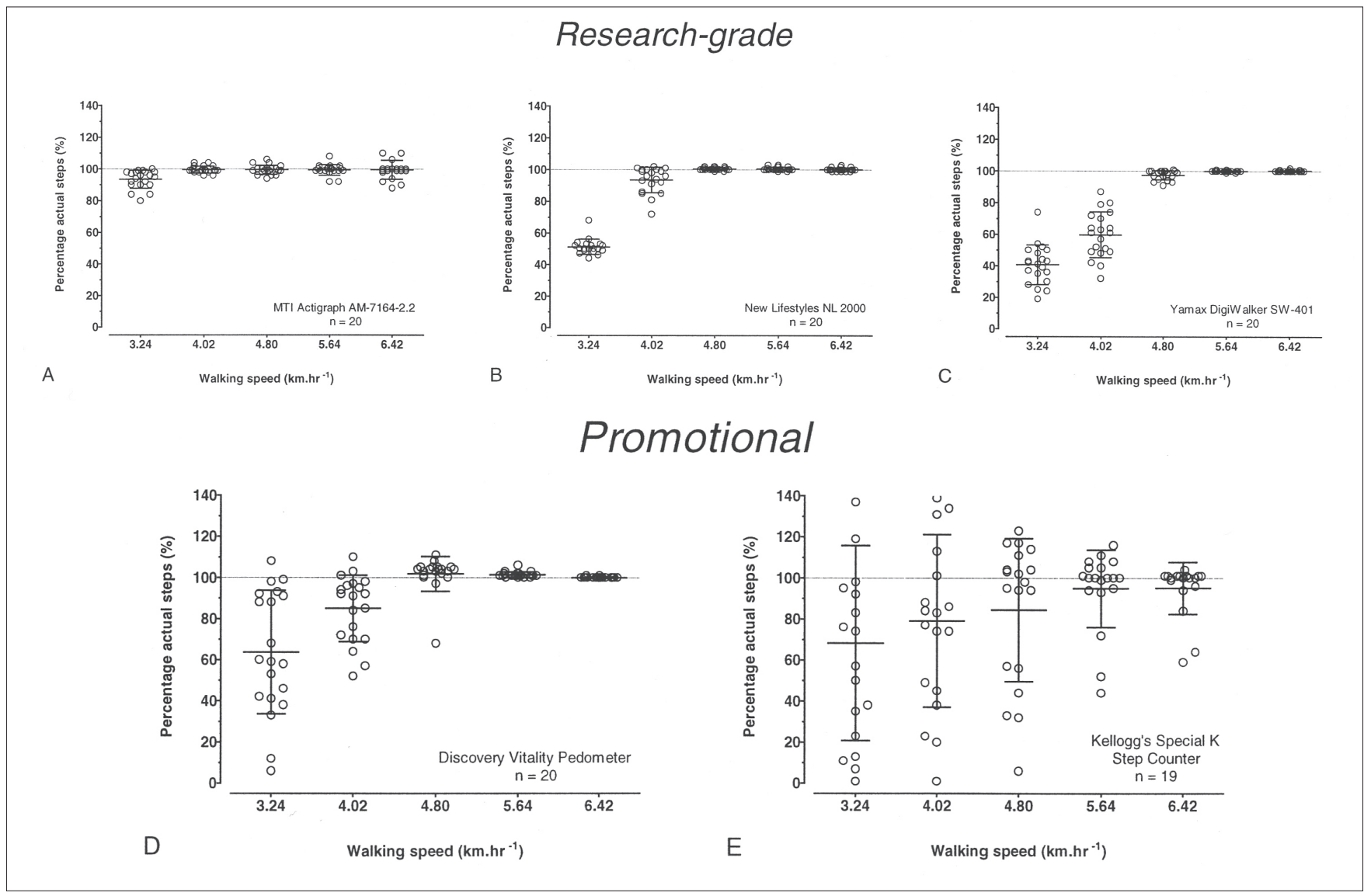

Fig. 2 A-E. Pedometer performance during treadmill walking (mean $\pm S D$ ).

\section{TABLE I. Output for various pedometer brands during ambulatory and non-ambulatory activities}

\begin{tabular}{|c|c|c|c|c|c|c|}
\hline \multirow[b]{2}{*}{$\begin{array}{l}\text { Pedometer brand }{ }^{1} \\
\text { Distance: } 62.9 \mathrm{~km}\end{array}$} & \multicolumn{5}{|c|}{ Treadmill walking (\% of actual steps $)^{2}$} & \multirow[t]{2}{*}{ Motor vehicle travel $\left(\text { steps. } \mathrm{km}^{-1}\right)^{3}$} \\
\hline & $3.24 \mathrm{~km} \cdot \mathrm{hr}^{-1}$ & $4.02 \mathrm{~km} \cdot \mathrm{hr}^{-1}$ & $4.80 \mathrm{~km} \cdot \mathrm{hr}^{-1}$ & $5.64 \mathrm{~km} \cdot \mathrm{hr}^{-1}$ & $6.42 \mathrm{~km} \cdot \mathrm{hr}^{-1}$ & \\
\hline \multicolumn{7}{|l|}{ Research-grade } \\
\hline MTI & $94(6)^{4,5}$ & $100(2)^{7}$ & $100(3)$ & $100(3)$ & $100(6)$ & $10.3(2.3)^{10}$ \\
\hline NL & $51(5)^{4}$ & $94(8)$ & $100(1)$ & $101(1)$ & $100(1)$ & $2.6(1.2)$ \\
\hline DW & $41(12)^{4,6}$ & $60(15)^{4,8}$ & $98(3)^{4}$ & $100(1)$ & $100(1)$ & $4.9(4.0)^{11}$ \\
\hline \multicolumn{7}{|l|}{ Promotional } \\
\hline $\mathrm{KL}$ & $68(48)$ & $79(42)$ & $84(35)^{9}$ & $95(19)$ & $95(13)$ & $2.2(11.3)$ \\
\hline VT & $64(30)^{4}$ & $85(16)^{4}$ & $102(9)$ & $101(1)$ & $100(0.3)$ & $0.7(1.3)$ \\
\hline \multicolumn{7}{|c|}{$\begin{array}{l}{ }^{1} \mathrm{MTI}=\mathrm{MTI} \text { Actigraph AM-7164-2.2, NL = New Lifestyles NL 2000, DW = Yamax DigiWalker SW-401, KL = Kellogg's Special K Step Counter, VT = Discovery Vitality Pedometer. } \\
\text { Data reported as }{ }^{2} \text { mean }(\mathrm{SD}) \text { and }{ }^{3} \text { median (interquartile range). } \\
{ }^{4} \text { Significantly different from all other speeds for the same pedometer brand }(p<0.02),{ }^{5} \mathrm{MTI}>\mathrm{NL}, \mathrm{DW}, \mathrm{KL}, \mathrm{VT}(\mathrm{p}<0.03),{ }^{6} \mathrm{DW}<\mathrm{KL}(\mathrm{p}<0.02),{ }^{7} \mathrm{MTI}>\mathrm{KL}(\mathrm{p}<0.04),{ }^{8} \mathrm{DW}<\mathrm{MTI}, \mathrm{NL}, \mathrm{VT} \\
(\mathrm{p}<0.003),{ }^{9} \mathrm{KL}<\mathrm{MTI}, \mathrm{NL}, \mathrm{VT}(\mathrm{p}<0.04),{ }^{10} \mathrm{MTI}>\mathrm{NL}, \mathrm{DW}, \mathrm{KL}, \mathrm{VT}(\mathrm{p}<0.05),{ }^{11} \mathrm{DW}>\mathrm{VT}(\mathrm{p}<0.01)\end{array}$} \\
\hline
\end{tabular}


TABLE II. Variance component analysis of the pedometer output obtained during treadmill walking

\begin{tabular}{|c|c|c|c|c|c|c|c|c|c|c|}
\hline \multirow{4}{*}{ Sources of variance } & \multicolumn{10}{|c|}{ Pedometer brand ${ }^{1}$} \\
\hline & \multicolumn{6}{|c|}{ Research-grade } & \multicolumn{4}{|c|}{ Promotional } \\
\hline & \multicolumn{2}{|l|}{ MTI } & \multicolumn{2}{|l|}{$\mathrm{NL}$} & \multicolumn{2}{|l|}{ DW } & \multicolumn{2}{|l|}{ VT } & \multicolumn{2}{|l|}{$\mathrm{KL}$} \\
\hline & Variance & $\%^{2}$ & Variance & $\%$ & Variance & $\%$ & Variance & $\%$ & Variance & $\%$ \\
\hline Inter-instrument & 0 & 0.0 & 1 & 0.2 & 11 & 1.3 & 53 & 10.4 & 759 & 60.4 \\
\hline Intra-instrument & 25 & 100 & 477 & 99.8 & 829 & 98.7 & 457 & 89.6 & 498 & 39.6 \\
\hline Walking trial & 6 & 25.7 & 460 & 96.2 & 765 & 91.0 & 263 & 51.6 & 107 & 8.5 \\
\hline Residual & 19 & 74.3 & 17 & 3.6 & 64 & 7.7 & 194 & 38.0 & 391 & 31.1 \\
\hline Total & 25 & 100 & 478 & 100 & 840 & 100 & 510 & 100 & 1257 & 100 \\
\hline
\end{tabular}

(DW, MTI, NL, VT) detected $\sim 100 \%$ of the actual steps (Fig. 2A-D, Table I). From 3.24 to $4.80 \mathrm{~km} \cdot \mathrm{hr}^{-1}$ pedometer output for the same brand differed significantly across speeds ( $p$ $<0.05$ ) (Table I). Below $5.64 \mathrm{~km} \cdot \mathrm{hr}^{-1}$, pedometers brands differed significantly over the same speed $(p<0.05)$ but not above $5.64 \mathrm{~km} . \mathrm{hr}^{-1}$ (Table I). Of particular note was the low inter-instrument variability of the MTI and NL pedometers even at low walking speeds (Fig. 2A-B).

From the variance component analysis (Table II), it is evident that the research-grade pedometers (DW, MTI, NL) have far less inter-instrument variability $(0 \%$ to $1.3 \%)$ than the VT pedometer (10.4\%) and especially the $\mathrm{KL}$ pedometer (60.4\%). Notably, the NL and MTI pedometers exhibited $\sim 0 \%$ inter-instrument variability and $\sim 100 \%$ intra-instrument variability (Table II). By far the lowest amount of variance for most of the variance components was found in the MTI pedometer (Table II). The walking trial and residual variation, nested within the intra-instrument variation, is also shown in Table II. Of the research-grade pedometers (DW, MTI, NL) the more sensitive MTI monitor demonstrated lower variation across the trials and higher residual variation, which is in contrast to the DW and NL monitors which displayed greater specificity (higher variation across trials and lower residual variation). Neither the KL nor the VT monitors quite reached the patterns of variability (walking speed, residual) of the research-grade pedometers (Table II).

The absolute per cent error for the DW, KL, MTI, NL and VT pedometers, at a treadmill walking speed of $4.8 \mathrm{~km} \cdot \mathrm{hr}^{-1}$, was $2.6(2.8) \%, 25.6(27.9) \%, 2.1(2.1) \%, 0.7(0.8) \%$ and 5.3 (6.8)\%, respectively. For a margin of error of $\pm 10 \%$, neither promotional pedometer achieved the accuracy levels of the research-grade pedometers (DW, MTI, NL: 100\% exact counting). Only the VT pedometer achieved the required standards of a promotional pedometer $(90 \%$ exact counts, $10 \%$ over-/undercounting). The $\mathrm{KL}$ pedometer exhibited poor accuracy levels (42.1\% exact counts, $57.9 \%$ over-/ undercounting).

\section{Part 2}

There were no pedometer failures during this experiment.
The distance, average speed and maximum speed for the motor vehicle trip was $62.9 \mathrm{~km}, 53.8$ (29.1) km.hr ${ }^{-1}$ and 121 $\mathrm{km} . \mathrm{hr}^{-1}$, respectively. With regard to placement of pedometers on the rig, there was no row $(F=1.900, p=0.200)$ or column ( $F=0.195, p=0.994)$ effect. In other words, whether the pedometer was mounted towards the back or front of the car, or mounted closer to the driver's side or passenger's side, did not have any effect on the pedometer output. The number of steps detected per kilometre differed significantly between some pedometers (Table I). The MTI detected significantly more steps than any of the other pedometers $(p<$ $0.05)$ and the DW detected significantly more steps than the VT $(p<0.01)$. The variability (interquartile range) of the $\mathrm{KL}$ pedometer was noticeably greater than that of any of the other pedometers (Fig. 3, Table I). In contrast, the NL had the lowest variability, and barring two outliers, the VT demonstrated relatively low variability and seemed relatively resistant to registering non-ambulatory signals as ambulatory signals (Fig. 3, Table I).

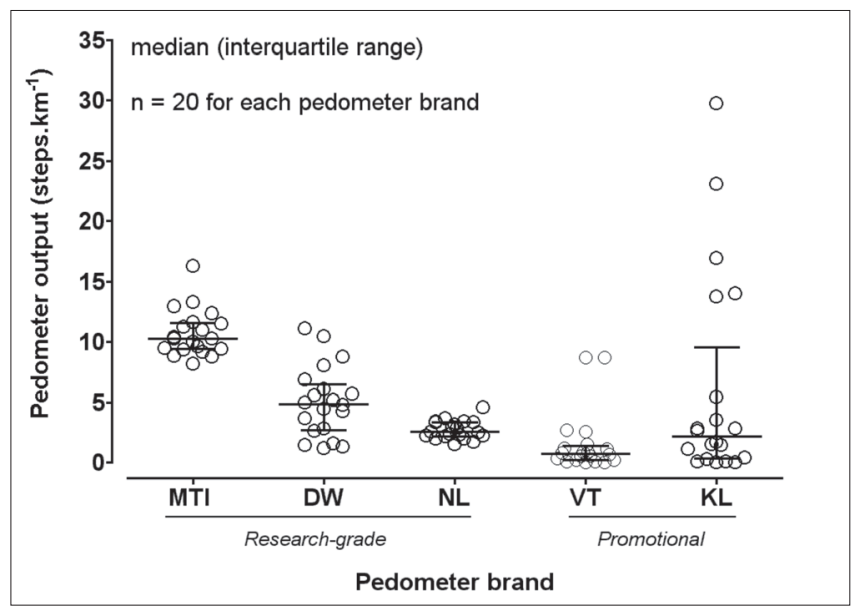

Fig. 3. Pedometer output in response to motor vehicle travel. $M T I=M T I$ Actigraph AM-7164-2.2, DW = Yamax DigiWalker SW-401, NL = New Lifestyles NL 2000, VT = Discovery Vitality Pedometer, KL = Kellogg's Special K Step Counter. 



\section{Discussion}

This study is novel in that it is the first study to report on the validity of pedometers, under controlled conditions, used in South African promotional programmes which emphasise physically active lifestyles. The principal findings of this study were firstly that the accuracy and quality of the $\mathrm{KL}$ pedometer was poor when compared with research-grade pedometers. Secondly, the KL pedometer was the most variable in its response to non-ambulatory signals, so much so that it would not be possible to determine a correction factor. Thirdly, neither the KL nor the VT pedometers quite reached a pattern of either high sensitivity (low walking trial variation, high residual variation) or high specificity (high walking trial variation, low residual variation) associated with researchgrade pedometers. Fourthly, the VT pedometer performed within the margin of error expected of promotional pedometers during validity testing under controlled conditions, but the $\mathrm{KL}$ pedometer did not, and should therefore not be used in promotional programmes.

The results from the present study are in agreement with the results from other pedometer calibration studies, specifically the greater sensitivity of accelerometer-type pedometers and the greater specificity of spring-type pedometers. ${ }^{5,8,9,21,22}$ Also in agreement with other work, the coiled spring DW pedometer was more variable than the piezo-electric $\mathrm{MTI}^{22}$ and $\mathrm{NL}^{8}$ pedometers, especially at lower walking speeds. Similarly, at lower walking speeds, hair-spring pedometers were more variable than the more accurate and durable coiled-spring pedometers. ${ }^{5}$ Finally, the results from this study concur that promotional pedometer output is particularly prone to inaccurate and erratic step counts. ${ }^{10,14,37}$

Studies have consistently demonstrated that for a level walking speed of $4.8 \mathrm{~km} \cdot \mathrm{hr}^{-1}$, most good quality pedometers should measure close to $100 \%$ accuracy., ${ }^{5,21}$ A margin of error from $\pm 1 \%{ }^{5,9,21,22}$ to less than $\pm 4 \%{ }^{25}$ has been found for research-grade pedometers during treadmill calibration at this speed. For verification tests using a fixed walking distance $^{32}$ or a fixed number of steps, ${ }^{40}$ the margin of error has ranged from $\pm 3 \%$ to $\pm 5 \%$. In a free-living environment, Schneider et al. ${ }^{31}$ suggest that in comparison to researchgrade pedometers, promotional pedometers should achieve at least $\mathrm{a} \pm 10 \%$ margin of error. Using the $\pm 10 \%$ margin of error for free-living environments, De Cocker et al. ${ }^{10}$ found that only $25.9 \%$ of the 973 promotional pedometers tested achieved this criterion.

A recent validation study from North America evaluated promotional pedometers that were distributed in cereal boxes (Kellogg's Special K). ${ }^{37}$ This study employed a novel testing battery that comprised a 20 -step test, a $4.8 \mathrm{~km} . \mathrm{hr}^{-1}$ treadmill walking test, a motor vehicle test for susceptibility to non-ambulatory signals and a 24-hour free-living test. The pedometers used as gold standard were the Yamax DigiWalker and MTI Actigraph. A novel aspect of the study was that it provided quantitative performance criteria by which to judge whether a pedometer is suitable for promotional or research purposes. ${ }^{37}$ Tudor-Locke et al. ${ }^{37}$ found that $53 \%$ of the promotional pedometers did not pass the 20-step test ( $>5 \%$ margin of error) compared to the $100 \%$ success rate of the DigiWalker. The mean absolute error for the treadmill walking test was 24.2 (33.9)\% for the Kellogg's pedometer in contrast to the $3.9(6.6) \%$ of the Digiwalker, ${ }^{37}$ which is similar to the results from the present study. The promotional pedometer detected 5.7-fold more steps during the motor vehicle test than the DigiWalker. ${ }^{37}$ In contrast, the present study found the DW to accumulate 2.2-fold more steps during the motor vehicle test than the $\mathrm{KL}$, although the $\mathrm{KL}$ variability was 2.8 -fold greater than the $\mathrm{DW}$, suggesting lower construction quality. The mean absolute per cent error under free-living conditions versus the Actigraph was 44.9 (34.5)\% for the Special K pedometer and 19.5 (21.2)\% for the DigiWalker. ${ }^{37}$ In the present study, the VT pedometer displayed lower absolute per cent error during treadmill walking and detected fewer steps during the motor vehicle ride, compared with the $\mathrm{KL}$ pedometer. However, it is important to note that although a pedometer can perform within the margin of error expected of promotional pedometers, it can demonstrate poor longevity, because of inferior construction methods and materials. Standardised tests and standards for judging appropriate longevity have yet to be developed.

The present study has also found the MTI to be more susceptible to non-ambulatory signals compared with the $\mathrm{DW}^{22}$ The difference in absolute values between this study and others, ${ }^{22,37}$ was likely the result of different mounting (human vs rig), vehicles (light motor vehicle vs recreational vehicle), vehicle speed and road surfaces. This study has also found that certain microprocessor algorithms, which exclude non-ambulatory signals, are less susceptible to nonambulatory signals. For instance, the NL and VT algorithms were better able to exclude non-ambulatory signals compared with the MTI algorithm. It should be noted that low-quality pedometer construction would negate any algorithm written to exclude non-ambulatory signals. Despite the MTI's higher false-positive count, the superior construction quality of the MTI pedometer would result in low inter-instrument variance such that a correction factor could be calculated. ${ }^{22}$ Similarly, even in the absence of signal-filtering algorithms, highquality coiled-spring pedometers (DW) are less variant than low-quality hair-spring pedometers $(\mathrm{KL})$ when responding to non-ambulatory signals, such that correction factors can be estimated. Moreover, the lower variability of the VT pedometer compared with the DW pedometer would be expected to reverse over time because of the inherent frailty of the hair spring used in the VT pedometer construction. ${ }^{24}$ This is despite the presence of a signal-filtering algorithm in the VT pedometer microprocessor.

The type of spring mechanism of lever-arm-type pedometers is essential to the accuracy and longevity of these instruments. Recent work has shown that hair-spring mechanisms fail substantially sooner than coil-spring mechanisms. ${ }^{24}$ In a novel study, McKenzie et al. ${ }^{24}$ tested one 

coil-spring pedometer (DigiWalker SW-701, $N=10$ ) and two hair-spring pedometers (Sportline Electronic 345, $N=10$ and McDonald's Stepometer, $N=10$ ) to failure after repeated bouts of 100000 steps on a customised bench-top orbital shaker at a simulated walking speed of $\sim 4.82 \mathrm{~km} . \mathrm{hr}^{-1}$. Failure was defined as $\geq 10 \%$ error for 50 steps at $4.82 \mathrm{~km} \cdot \mathrm{hr}^{-1}$ on a motorised treadmill. Only new pedometers, that measured accurately to $<10 \%$ prior to testing, were evaluated. The DigiWalkers did not fail and the testing protocol was discontinued at 2.38 million steps because of high-step accumulation. The Sportline and McDonald's pedometers failed at 710000 steps and 250000 steps, respectively. ${ }^{24}$ Assuming a sedentary individual accumulates 5000 steps. day $^{-1}$, the Sportline and McDonald's pedometers would measure accurately for 142 days and 50 days, respectively. For an active individual, accumulating 10000 steps.day $^{-1}$, the Sportline and McDonald's pedometers would measure accurately for 71 days and 25 days, respectively. Because of the similarity between the mechanism and quality of construction of the Kellogg's and McDonald's pedometers, and the Sportline and Discovery Vitality pedometers it would seem reasonable to expect similar failure rates for the promotional pedometers tested in the present study. These findings strongly suggest that prior to any promotional programme incorporating pedometers, longevity tests should be conducted either by the manufacturer or independent research institutions.

Users of the VT should be aware that the accreditation that appears on the packaging of the pedometer refers to the Vitality programme and not to the quality of the pedometer. In other words, the pedometer has not been tested for validity or longevity by any accrediting agency of the Discovery Vitality Programme (personal communication, Laurence Rau, Vitality Programme, Discovery Health, SA). Similarly, there is no indication that the validity or longevity of the $\mathrm{KL}$ pedometer has been tested either by Kellogg's South Africa or an independent institution. It is important to note that, unlike the DW and NL pedometers, neither the $\mathrm{KL}$ nor VT pedometers include in their instruction sheets or manuals any directions for simple tests that can be carried out by the user of the pedometer to determine if the pedometer is measuring accurately. For instance, in the DW instruction sheet, directions are provided for a simple 20-step test that the user can implement to determine if the pedometer is relatively accurate.

It has been suggested that the accuracy of the VT pedometer is not particularly essential and that an expensive pedometer brand would limit people's involvement in the Vitality pedometer initiative. Rather, the educational value of the Vitality pedometer initiative as a whole is more important (personal communication, Laurence Rau, Vitality Programme, Discovery Health, SA). However, the reason pedometers are used is the direct, immediate and easily interpretable feedback they supply through the step count. ${ }^{35}$ Moreover, pedometers serve as a memory prompt and reminder with regard to maintaining physical activity behaviours. ${ }^{38}$
Villanova et al. ${ }^{39}$ have noted that pedometers can provide a consistent, quantitative measure, a precise objective and can positively influence self-efficacy. Consequently if the output is inaccurate and variable, the feedback is no longer reliable and cannot positively influence self-efficacy. The loss of positive feedback could theoretically adversely affect behaviour modification or adherence, defeating the rationale of the Vitality 'points' system. With regard to cost, a viable alternative to the VT ( 14 USD assuming an exchange rate of 7 ZAR to 1 USD) would be the DW pedometer which costs 20-30 USD. The Australian 10000 Steps Programme utilises the DW pedometer and discounts are given for bulk orders. ${ }^{1}$ There are reports that a pedometer promotion run by Coca Cola in Florida, USA had a pedometer return rate exceeding $60 \%$ because of product failure and inaccuracy (personal communication, Michael Cordier, National Sales Director, Walk4Life Inc., USA). It is likely then that a significant number of promotional pedometers would probably either cease to be used or be returned because of inaccuracy or failure. The higher cost of the DW would be offset by fewer returns of new instruments and far greater durability of existing instruments. Companies and organisations should investigate the most cost-effective strategy (cheap, lowquality pedometers with high failure rates vs. more expensive, high-quality pedometers with low failure rates) and whether they are committed to providing a pedometer brand which is more likely to favourably affect behaviour modification or adherence in programme participants. An alternative strategy would be to distribute better quality pedometers in a more selective manner to targeted groups or neighbourhoods. ${ }^{14}$

Kellogg's and Discovery Health are not the only South African companies that have launched programmes promoting a physically active lifestyle by distributing pedometers. Unilever launched the Flora Comrades Step Counter (FL) promotion which started in time for the 2005 Flora Comrades Marathon. Unfortunately, samples of these pedometers could not be obtained in time for inclusion in the study. However, a sample pedometer was recently obtained and upon inspection it was ascertained that the step-sensing mechanism consists of a lever arm, hair-spring mechanism, and a magnet on the end of the lever arm which activates a reed switch, completing the electric circuit and recording one step (Fig. 1). Furthermore, the FL pedometer does not contain an algorithm to filter out non-ambulatory signals. The control buttons are not covered so the unit could be inadvertently reset. On further inspection, the quality of manufacture of the FL pedometer is similar to that of the $\mathrm{KL}$ and VT pedometers. Based on the spring-mechanism type and the quality of construction, it would seem reasonable to conclude that the FL pedometer would not have performed any better than the VT pedometer, and would most likely have performed similarly to the $\mathrm{KL}$ pedometer. There is no indication that the validity or longevity of the FL pedometer has been evaluated by Unilever, nor are there any published data to that effect.

This study does not address the effects of different 
levels of body weight and fat distribution, ${ }^{8}$ pedometer tilt ${ }^{8}$ and pedometer placement ${ }^{5}$ on pedometer accuracy. Further studies are required to test the promotional pedometers presented in this study under free-living conditions ${ }^{10,31,37}$ and to assess the 'longevity' 24 of the units. Are the results of this investigation generalisable to free-living conditions? Le Masurier et al. ${ }^{21}$ have shown that if a pedometer performs poorly in the laboratory it will probably perform poorly in free-living conditions, suggesting that tests under controlled conditions are generalisable to free-living conditions. Controlled testing conditions allow the investigation of intensity- or speed-dependent responses of pedometers thus quantifying the sensitivity or specificity of the units. ${ }^{21}$ In other words, are pedometers sensitive to a range of intensities (low-to-vigorous) or are pedometers specific to certain intensities (moderate-to-vigorous)? Consequently, pedometers that demonstrate high sensitivity are susceptible to non-ambulatory signals ${ }^{22}$ while pedometers that demonstrate high specificity will not detect steps accurately in populations that naturally ambulate at slower speeds, for example the elderly and those with a shuffling gait. ${ }^{9,25}$ Moreover, correction factors for pedometer output due to motor vehicle travel determined under controlled conditions ${ }^{22}$ have been used in later free-living studies. ${ }^{21}$ Free-living conditions will however highlight problems relating to the inadvertent stopping or resetting of the unit because control buttons are not covered, ${ }^{10}$ and the amount of misclassification of the physical activity status in relation to public health guidelines. ${ }^{34}$

In conclusion, this investigation has shown that data from promotional pedometers must be treated with caution. In order for users to obtain reasonably valid and reliable readings from pedometers, companies or organisations initiating promotional programmes should consider the following (listed in order of preference, can include more than one recommendation as a verification procedure):

- use a more expensive, but valid and durable pedometer, such as the Yamax DigiWalker 5,6,9,21,24,32

- require some form of quality control for validity and longevity from the manufacturer or an independent institution. For instance, the Japanese Industrial Standard requires that pedometers achieve an error level of less than $\pm 3 \%$ (3 steps out of 100 steps). ${ }^{31}$ Standardised tests, using a research-grade pedometer as a standard, could include performance under free-living conditions, ${ }^{10}$ laboratory trials of level walking, ${ }^{5,14}$ susceptibility to non-ambulatory signals (motor vehicle), ${ }^{22,37}$ and longevity tests or 'tests to failure'. ${ }^{24}$ A comprehensive yet easily implemented protocol, which includes testing under controlled and free-living conditions, has recently been proposed. ${ }^{37}$

- have a simple mechanical calibration device located at central points to be used prior to distributing the pedometers and at regular intervals during use. Those pedometers performing outside certain limits should be replaced.

- Include self-calibration or self-verfication procedures in the instructions, which should be performed periodically to ver- ify accuracy. Those pedometers performing outside certain limits should be replaced.

- a simple 'shake test' which requires the user to shake the pedometer up and down 100 times. ${ }^{40}$ The reading must be within $\pm 5 \%$ (95 - 105 steps)

- a 20-step walking test. ${ }^{37,40}$ The reading must be within $\pm 5 \%$ (19- 21 steps $)$

- a 50 -step ${ }^{24}$ or $100-$ step $^{14,40}$ walk on a level, firm surface at a moderate pace $\left(4.80 \mathrm{~km} . \mathrm{hr}^{-1}, 3.3 \mathrm{METs}, 50 \mathrm{~m}\right.$ in $37.5 \mathrm{~s}$ or $100 \mathrm{~m}$ in $1 \mathrm{~min} 15 \mathrm{~s}$ ) repeated twice and the average taken. This should be done with the pedometer on the dominant side of the body. At this speed research-grade pedometers should be $\pm 1 \%$ accurate. . $^{5,21}$ Acceptable accuracy levels for promotional pedometers should be $<10 \%$ ( \pm 4 steps of 50 steps or \pm 9 steps of 100 steps). ${ }^{37}$

\section{Acknowledgements}

The Research Development and Administration Division of the University of Limpopo (Turfloop Campus), and the Institutional Research Development Programme of the National Research Foundation supported this study.

\section{REFERENCES}

1. 10000 Steps Programme (Australia). Available at: http://www.10000steps. org.au. Accessed August 2006.

2. Ainsworth BE, Haskell WL, Whitt MC, et al. Compendium of physical activities: an update of activity codes and MET intensities. Med Sci Sports Exerc 2000; 32: S498-S504.

3. America on the Move. Available at: http://pedometer.americaonthemove. org/store/detail.asp?id=1. Accessed August 2006.

4. Anjos LA, Wahrlich V, Vasconcellos MTL. Distribution of pedometer count in a population-based sample of adults from Niterio, Rio de Janerio, Brazil. Med Sci Sports Exerc 2005; 37: S324.

5. Bassett DR, Ainsworth BE, Leggett SR, Mathien CA, Main JA, Hunter DC, Duncan GE. Accuracy of five electronic pedometers for measuring distance walked. Med Sci Sports Exerc 1996; 28: 1071-77.

6. Bassett DR, Strath SJ. Use of pedometers to assess physical activity. In: Welk GJ, ed. Physical Activity Assessments for Health-related Research. Champaign, IL: Human Kinetics, 2002: 163-77.

7. Brage S, Brage N, Wedderkopp N, Froberg K. Reliability and validity of the Computer Science and Applications accelerometer in a mechanical setting. Meas Phys Educ Exerc Sci 2003; 7: 101-19.

8. Crouter SE, Schneider PL, Bassett DR. Spring-levered versus piezo-electric pedometer accuracy in overweight and obese adults. Med Sci Sports Exerc 2005; 37: 1673-9.

9. Crouter SE, Schneider PL, Karabulut M, Bassett DR. Validity of 10 electronic pedometers for measuring steps, distance, and energy cost. Med Sci Sports Exerc 2003; 35: 1455-60.

10. De Cocker K, Cardon G, De Bourdeaudhuij I. Validity of the inexpensive Stepping Meter in counting steps in free living conditions: a pilot study. $\mathrm{Br}$ J Sports Med 2006; 40: 714-6.

11. Digital Planet: Discovery Vitality Pedometer (Customer Reviews). Available at: http://www.digitalplanet.co.za/shop/product.asp?stockID=59886. Accessed August 2006.

12. Discovery Health. Available at: http://www.discovery.co.za. Accessed August 2006 .

13. Doheny, K. Pedometers tested as step to more exercise: McDonald's, others try a simple device to get people moving. Available at: http://www. healthscout.com/news/407/515213/main.html. Accessed August 2006.

14. Gao $Y$, Boscolo M, Krahling H, Zhu W, Lee M. Step-count accuracy and instrument equivalence of McDonalds' Stepometers. Med Sci Sports EXerc 2005; 37: S117. 
15. James PT, Leach R, Kalamara E, Shayeghi M. The worldwide obesity epidemic. Obes Res 2005; 9: 233S-88S.

16. Jordan AN, Jurca GM, Tudor-Locke C, Church TS, Blair SN. Pedomete indices for weekly physical activity recommendations in postmenopausa women. Med Sci Sports Exerc 2005; 37: 1627-32.

17. Kearney PM, Whelton M, Reynolds K, Muntner P, Whelton PK, He J. Global burden of hypertension: analysis of worldwide data. Lancet 2005 365: 217-23.

18. Kellogg's Special K 10000 Step Challenge (United Kingdom). Available at: http://www.kellogs.co.uk. Accessed April 2006.

19. Kellogg's Special K 2000 Steps Challenge (South Africa). Available at: http://specialk.experthealth.co.za/live/index.php. Accessed August 2006.

20. Kirk AF, Higgins LA, Hughes AR, Fisher BM, Mutrie N, Hillis S, Maclntyre PD. A randomized, controlled trial to study the effect of exercise consultation on the promotion of physical activity in people with Type 2 diabetes: a pilot study. Diabet Med 2001; 18: 877-82.

21. Le Masurier GC, Lee SM, Tudor-Locke C. Motion sensor accuracy under controlled and free-living conditions. Med Sci Sports Exerc 2004; 36: $905-$ 10.

22. Le Masurier GC, Tudor-Locke C. Comparison of pedometer and accelerometer accuracy under controlled conditions. Med Sci Sports Exerc 2003; 35: $867-71$.

23. Matthews CE, Ainsworth BE, Thompson RW, Bassett DR. Sources of variance in daily physical activity levels as measured by an accelerometer Med Sci Sports Exerc 2002; 34: 1376-81.

24. McKenzie J, Manning T, Juergens CA, Heil DP. A lab-test to evaluate pedometer longevity. Med Sci Sports Exerc 2005; 37: S117.

25. Melanson EL, Knoll JR, Bell ML, Donahoo WT, Hill JO, Nysse LJ, Lanningham-Foster L, Peters JC, Levine JA. Commercially available pedometers: considerations for accurate step counting. Prev Med 2004; 39: 361-8.

26. Metcalf BS, Curnow JS, Evans C, Voss LD, Wilkin TJ. Technical reliability of the CSA activity monitor: The EarlyBird Study. Med Sci Sports Exerc 2002; 34: 1533-7.

27. Moreau KL, Degarmo R, Langley J, McMahon C, Howley ET, Bassett DR Thompson DL. Increasing daily walking lowers blood pressure in postmenopausal women. Med Sci Sports Exerc 2001; 33: 1825-31.

28. Patterson RE, Frank LL, Kristal AR, White E. A comprehensive examination of health conditions associated with obesity in older adults. Am J Prev Med 2004; 27: 385-90.

29. Pett MA. Nonparametric Statistics for Health Care Research. Thousand Oaks, CA: Sage Publications, 1997.

30. Puoane T, Steyn K, Bradshaw D, Laubscher R, Fourie J, Lambert V Mbananga N. Obesity in South Africa: The South African Demographic and Health Survey. Obes Res 2002; 10: 1038-48.
31. Schneider PL, Crouter SE, Bassett DR. Pedometer measures of freeliving physical activity: comparison of 13 models. Med Sci Sports Exerc 2004; 36: 331-5

32. Schneider PL, Crouter SE, Lukajic O, Bassett DR. Accuracy and reliability of 10 pedometers for measuring steps over a 400-m walk. Med Sci Sports Exerc 2003; 35: 1779-84

33. The Anatomy of a Pedometer. Available at: http://www.pedometers.com. Accessed August 2006

34. Tudor-Locke C, Bassett DR. How many steps/day are enough? Preliminary pedometer indices for public health. Sports Med 2004; 34: 1-8.

35. Tudor-Locke CE, Myers AM. Methodological considerations for researchers and practitioners using pedometers to measure physical (ambulatory) activity. Res Q Exerc Sport 2001; 72: 1-12.

36. Tudor-Locke C, Lind KA, Reis JP, Ainsworth BE, Macera CA. A Preliminary evaluation of a pedometer-assessed physical activity self-monitoring survey. Field Methods 2004; 16: 422-38

37. Tudor-Locke C, Sisson SB, Lee SM, Craig CL, Plotnikoff RC, Bauman A. Evaluation of quality of commercial pedometers. Can J Public Health 2006; 97: S10-5.

38. Tudor-Locke CE, Myers AM, Bell RC, Harris SB, Wilson Rodger N. Preliminary outcome evaluation of the First Step Program: a daily physical activity intervention for individuals with type 2 diabetes. Patient Educ Couns 2002; 47: 23-8.

39. Villanova N, Pasqui F, Burzacchini S, et al. A physical activity program to reinforce weight maintenance following a behavior program in overweight/ obese subjects. Int J Obes 2006; 30: 697-703.

40. Vincent SD, Sidman CL. Determining measurement error in digital pedometers. Meas Phys Educ Exerc Sci 2003; 7: 19-24

41. Walking the Way to Health Initiative. Available at: http://www.whi.org.uk. Accessed August 2006

42. Wallis C. The obesity warlords. Time 2004; 164: 46-51.

43. Welk GJ. Physical Activity Assessments for Health-Related Research. Champaign, IL: Human Kinetics, 2002.

44. Welk GJ. Use of accelerometry-based activity monitors to assess physical activity. In: Welk GJ, ed. Physical Activity Assessments for Health-Related Research. Champaign, IL: Human Kinetics, 2002: 125-41.

45. Wild S, Roglic G, Green A, Sicree R, King H. Global prevalence of diabetes. Estimates for the year 2000 and projections for 2030. Diabetes Care 2004; 27: 1047-53.

46. Yach $D$, McKee M, Lopez AD, Novotny $T$, for O, V. Improving diet and physical activity: 12 lessons from controlling tobacco smoking. BMJ 2005; 330: 898-900.

47. Yusuf $\mathrm{S}$, Vaz M, Pais P. Tackling the challenge of cardiovascular disease burden in developing countries. Am Heart J 2004; 148: 1-4. 